\title{
A QoS-aware MAC protocol for Wireless Sensor Networks
}

\author{
Guoqiang Zheng ${ }^{1,2}$, Yaru Sun ${ }^{1}$, Bingwu Kang ${ }^{1}$, Huahong $\mathrm{Ma}^{1}$, Jishun $\mathrm{Li}^{2,3}$ and Yuting Wang ${ }^{1}$ \\ ${ }^{1}$ College of Information Engineering, Henan University of Science and Technology \\ Luoyang 471023, China \\ ${ }^{2}$ Henan Key Laboratory for Machinery Design and Transmission System, Henan University of Science and Technology \\ Luoyang 471003, China \\ ${ }^{3}$ State Key Laboratory of Mining Heavy Equipment \\ Luoyang 471039, China
}

\begin{abstract}
Considering traditional MAC protocols for Wireless Sensor Networks cannot provide service differentiation for different traffic classes, we propose a Self-adaption QoS-aware MAC protocol (SQ-MAC). The protocol can provide collision-free time slots for nodes, and take different contention window size for different traffic classes. It can also introduce virtual multiqueue and relatively fair scheduling mechanism for data packets. Simulation results show that, compared with the low priority traffic, the transmission quality of high priority traffic is effectively improved, in terms of packet delivery ratio, latency and so on. Additionally, compared with S-MAC and DQ-MAC, SQ-MAC also maintains a good network performance.
\end{abstract}

Keywords: Wireless Sensor Networks, service differentiation, QoS-aware MAC.

\section{Introduction}

Wireless Sensor Networks (WSN) is a multi-hop and selforganizing network system, it's composed of a large number of tiny sensor nodes, which is formed through wireless communication [1]. With the development of science and technology, the related technologies of WSN become more and more mature. Simple data acquired by WSN have been unable to meet the comprehensive needs of people. The traffic class diversification has become the development direction. However, the traditional Media Access Control (MAC) protocols are assumed to use a single Best Effort (BE) data transfer model, a variety of traffic classes fairly competing network resources, not conducive to the higher quality of service (QoS) requirements of some network applications. So, it has a relatively positive significance to research the QoS-aware MAC protocols [2].

MAC protocols for WSN can be divided into scheduling class, competitive class and hybrid class according to the channel access modes [3]. Literature [4] presented a QoSaware MAC protocol based on competition, which can adapt well to the changing network conditions. While, there is no synchronization between nodes, which is likely to introduce serious problems including idle listening and early sleeping. Additionally, the protocol uses an unfair packet scheduling, causing that the lower priority packets suffer from higher latencies. The main idea of Literature [5] is that, based on service differentiation, an additional channel listening time was introduced for high priority traffic. As a result, compared to the low priority traffic, the high priority traffic can achieve more chance to be delivered. Although the protocol improves high priority traffic's network performance, it doesn't consider the increasing latency and energy consumption caused by data collision and idle listening.

Literature [6] presented a QoS-aware MAC protocol based on scheduling, which can bound latency and reliability of data. However, it is easy to cause synchronization errors accumulation due to each node synchronizes its clock with its parent node, thus it does not scale well for large WSN. Literature [7] presented M-ASAP based on TDMA, it makes data section and control section cleverly designed in a crossed style, and the binary tree method is adopted in the slot allocation algorithm. M-ASAP avoids the jitter between various time slots, and achieves QoS-aware according to slot allocation algorithm. However, it increases extra latency and energy consumption caused by binary tree when the network load is low.

Literature [8] presented a hybrid QoS-aware MAC protocol, I-MAC. Although I-MAC combines the strength of both TDMA and CSMA, it still needs tight clock synchronization which is a well-known drawback of TDMA. Additionally, possessing up-to-date neighbor information and slot schedule in highly dynamic networks is a great challenge. Literature [9] also presented a hybrid MAC protocol. It makes the nodes dynamically form the priority table of time slots according to traffic classes of nodes, and competing for these time slots. Simulation results show that, the MAC protocol can assure QoS of 
data, while its scalability is terrible because the MAC parameters are fixed.

Energy consumption is the primary consideration factor for WSN, so MAC protocols not only meet the requirements of related applications, but also consider energy consumption of network. S-MAC is the first MAC protocol for the energy saving requirements of WSN, which reduces idle listening by using the duty cycle mechanism, eliminates overhearing and guarantee the data integrity by using RTS/CTS/DATE/ACK data exchange mechanism, reduces the latency of multi-hop transmission by using the adaptive listening $[10,11]$.

On the basis of S-MAC, we propose a QoS-aware MAC protocol, SQ-MAC. SQ-MAC realizes collision-free transmission by exchanging control frames, and makes the high priority traffic has more time slots for data transfer. Additionally, it uses adaptive back-off mechanism to provide different contention window $(\mathrm{CW})$ for the different priority traffic classes, and uses virtual multi-queue and fair scheduling mechanism to ensure QoS of all traffic classes. Thus, the high priority traffic will consume more network resources than the low priority traffic.

The rest of the paper is organized as follows. In Section 2, the SQ-MAC is described. In Section 3, we present the analytical model of SQ-MAC, while the simulation results are shown and discussed in Section 4. Finally, we conclude the work in Section 5.

\section{SQ-MAC Design}

SQ-MAC is a kind of QoS-aware MAC protocol for network applications with multi traffic classes, which uses periodic wake mechanism, as shown in Fig. 1, including synchronization phase, scheduling phase and data phase.

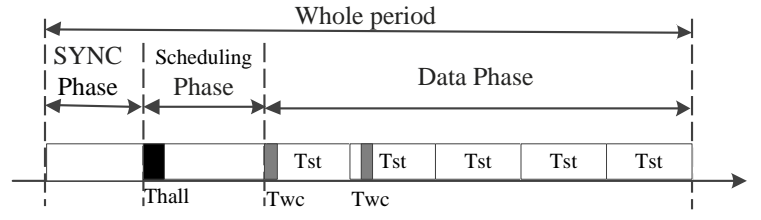

Fig. 1: Periodic wake mechanism of SQ-MAC.

In synchronization phase, similar to S-MAC, the virtual cluster is formed by the exchange of the synchronous frame first. To maintain time synchronization of the same virtual cluster, all nodes need to regularly broadcast their own scheduling information.

In scheduling phase, the sender and the corresponding receiver are required to complete the time slot scheduling by exchanging the RTSn/CTSn control frame, as shown in Fig. 2, including the selected time slot. The number of time slots is set to $\mathrm{S}$, it's determined by network nodes density, and the length of scheduling phase is proportional to $\mathrm{S}$. When network load is light, the nodes do not need to keep idle listening for the entire scheduling phase. So a time threshold is defined, Thall, which is the maximum time of nodes transmission RTSn in the ideal condition (no data collision) . If the duration time of channel being idle longer than Thall, unrelated nodes will turn to sleep.

The data phase is divided into a series of same length time slots, so the time length of data phase is equal to $S$ multiply by $\mathrm{T}_{\mathrm{st}}$, where $\mathrm{S}$ is time slots number, $\mathrm{T}_{\mathrm{st}}$ is the time length of single time slot. In theory, only one pair of nodes can exchange data in each time slot, so $\mathrm{T}_{\mathrm{st}}$ is equal to the time length of a whole RTS/CTS/DATE/ACK data exchange in the ideal condition.

The sender will be awaken for data transmission in the scheduled time slot. If the fading channel or data collision show up, time slots scheduling may fail, so a time threshold is defined, TWC, which is the time of nodes transmission RTS/CTS in ideal condition. The waiting time of nodes waiting for data packets and RTS control frame is TWC.

\begin{tabular}{|c|c|c|c|c|c|c|}
\hline type & size & $\begin{array}{l}\text { source } \\
\text { address }\end{array}$ & $\begin{array}{l}\text { destination } \\
\text { address }\end{array}$ & duration & CRC & \\
\hline \multicolumn{7}{|c|}{ Control frame of RTS/CTS } \\
\hline type & size & $\begin{array}{l}\text { source } \\
\text { address }\end{array}$ & $\begin{array}{l}\text { destination } \\
\text { address }\end{array}$ & duration & Time slot & CRC \\
\hline
\end{tabular}

Fig. 2: Structure of RTSn/CTSn.

In this paper, we have two traffic classes which are RT (Real-time) and NRT (Non-real-time), in the same sequence as their priorities. RT has higher QoS requirements on latency and higher packet delivery, which is used to send audio, video, etc. While, NRT has lower QoS requirements on latency and high packet delivery, which is used to transmit text, pictures, etc. SQ-MAC can achieve QoS-aware function through realizing service differentiation of two traffic classes [12, 13].

\subsection{Slot-scheduling Mechanism}

The slot-scheduling mechanism is that, nodes can schedule time slots by exchanging RTSn/CTSn control frame in scheduling phase. After completing time slots scheduling, only one pair of nodes exchange data in corresponding time slot, which can eliminate data collision in theory.

The performance indicators of latency and packet delivery rate will be improved if the traffic class has more time slots. As shown in Fig. 3, SQ-MAC divides all time slots of data 
phase into two parts. The first part are only allocated to RT, while both RT and NRT can use the second part. It means RT can use time slots' number is S, while NRT can only use time slots' number is $\mathrm{C}(\mathrm{S}>\mathrm{C})$. Based on the relevant requirements of network applications, SQ-MAC determines the time slots' proportions about different traffic classes.

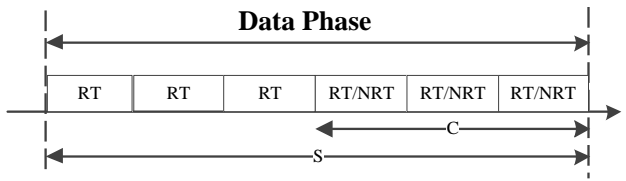

Fig. 3: Division of time slots in data phase.

The briefly process of slot-scheduling mechanism is as follows. In scheduling phase, the time slot number will be initialized to $X$, which is the first available time slot number of different traffic classes, Thus, the range of nodes scheduling time slots number is $[\mathrm{X}, \mathrm{S}]$. During scheduling phase, nodes need to make some judgments if they have listened the unrelated RTSn/CTSn, which are as follows:

- Node checks the recording address information of control frame. If source node or destination node is its own destination node, the node will cancel the time slot scheduling.

- Node checks the recording destination node of control frame, whether it belongs to the same virtual cluster. If it is, according to the control frame, node will remove the time slot number which cannot be scheduled. If the current time slot can be scheduled is 0 , the node will cancel the time slot scheduling.

After nodes successfully access channel, it will schedule the smallest time slot in all available slots, And the nodes which don't need to transmit data will send corresponding control frame, CTSn base on the receiving RTSn.

\subsection{Adaptive Back-off Mechanism}

For CSMA/CA mechanism, nodes need to use random back-off in the process of channel contention. The probability of nodes access channel is mainly effected by the $\mathrm{CW}$ size, that is, $\mathrm{CW}$ is more smaller, node access channel is more easier [14, 15]. SQ-MAC can achieve service differentiation by setting different $\mathrm{CW}$ size for different priority traffic classes. In addition, the WSN data generally are sudden, which requires MAC protocols to have good dynamic adaptability. In scheduling phase, an adaptive back-off mechanism is proposed for different traffic classes. Considering the time length of scheduling phase is limited, during the back-off process, nodes do not reset their back-off timer but temporarily freeze the timer if they have listened the unrelated RTSn/CTSn. After analyzing slot-scheduling mechanism, nodes can determine whether to contend channel before the scheduling phase is over.

Step1: $C W c u r=[C W m a x+C W m i n / 2]$

Step2: Observe the result of RTSn/CTSn transmit

Step3: If RTSn Success

Step4:CWmod =int [rand[RTdown, 1]]*(CWmin-CWcur $)]$ CWmod=int [rand[0,NRTdown]] *(CWmin-CWcur $)]$

Step5: If RTSn Fail

Step6: $C W m o d=\operatorname{int}[$ rand [0,RTup]] $*(C W m a x-C W c u r)]$ CWmod $=$ int [rand [NRTup, 1]] $*(C W m a x-C W c u r)]$

Step7: $C W c u r=C W c u r+C W m o d$

Fig. 4: Algorithm of adaptive CW for channel contention

The algorithm pseudo-code is shown in Fig. 4, where $\mathrm{CW}$ cur is the current $\mathrm{CW}$ value, CWmax is the maximum $\mathrm{CW}$ value, $\mathrm{CWmin}$ is the minimum $\mathrm{CW}$ value, and CWmod is the modified CW value. Nodes dynamically adjust CWcur size by observing the transmission result of RTSn in real-time. RTdown and NRTdown are the decreasing factors of $\mathrm{CW}$ size, while RTup and NRTup are the increasing factors of $\mathrm{CW}$ size. Because $0<$ RTup $<$ NRTup $<1$ and $1>$ RTdown $>$ NRTdown $>0$, SQMAC increases $\mathrm{CW}$ size faster for lower priority traffic, on the other hand, decreases $\mathrm{CW}$ size faster for higher priority traffic. RT always has a relatively high probability of access channel when compared with NRT.

\subsection{Virtual Multi-queue and Fair Scheduling Mechanism}

MAC protocols that employ service differentiation to classify the carried traffic class into different priority levels and the MAC protocol either maintaining a single queue for every traffic class or separating queues for each of them. As shown in Fig. 5(a), main drawback of the singlequeue scheme is the high cost of managing relatively long data queue. Since different priority packets are stored in the same queue, it is impractical to keep them sorted and process the packets according to their priorities. While, as shown in Fig. 5(b), the multi-queue scheme chops the long single queue into pieces and employs smaller different priority queues. In this way, packets can be served with a simple FIFO fashion for each priority and additional sorting or searching operations are not needed [16].

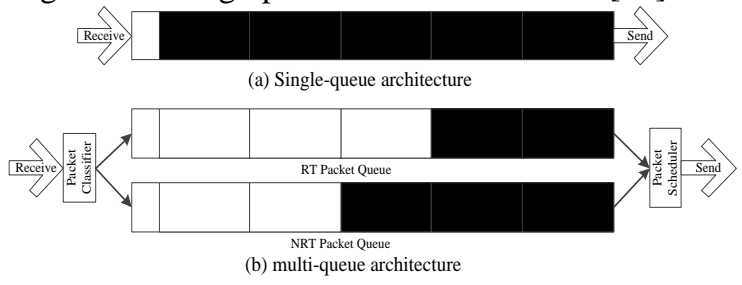

Fig. 5: Queue architecture of data scheduler. 
However, if the higher priority queue is always before the lower priority queue explicitly, there is possibility of intolerable performance for lower priority traffic in terms of latency and packet delivery ratio. A fair scheduling mechanism is proposed, where each traffic class has its own weight value by recording the specific time of data packets into queue. Packet scheduling of SQ-MAC selects the next serviced packet based on weights of data packets.

\section{Step1: Weightcur $=D W^{*} I T$ \\ Step2: Check the weight of first packet in each queue \\ Step3: Send the minimum weight of data packet}

Fig.6: Algorithm of fair scheduling for data

The pseudo-code is shown in Fig. 6, where IT is the time of data packets coming into the data queue, DW is the default variable of weight, Weightcur is the weight of data packets. Because DW(NRT) $>$ DW(RT), SQ-MAC can ensure the NRTs' QoS requirements while providing service differentiation.

\section{Analytical Model Of SQ-MAC}

In this section, we research the QoS-aware function according to analyze packet delivery ratio, latency and energy consumption in theory.

\subsection{Packet Delivery Ratio}

SQ-MAC realizes collision-free data exchange in data phase, so we analyze the packet delivery ratio by analyzing random back-off of scheduling phase.

Assuming the sensor nodes' number within the network is w, network is saturated, which means that there are data packets to be sent in every data queue. This paper uses random variable, $a(t)$ repesents the current size of $\mathrm{CW}, \mathrm{b}(\mathrm{t})$ is the current value of $\mathrm{CW}$ at moment $\mathrm{t}$. So as to construct a two-dimensional discrete time Markov chain, $\{\mathrm{a}(\mathrm{t}), \mathrm{b}(\mathrm{t})\}$. The remaining parameters are defined as follows:

- $\quad \mathrm{k}$ : the current value of $\mathrm{CW}$;

- $\quad$ : : the size of $\mathrm{CW}$ after a random variation, which is an uncertain number;

- $\mathrm{d}$ : the times of $\mathrm{CW}$ random changes, which is an uncertain number;

- $\mathrm{m}$ : maximum size of $\mathrm{CW}, \mathrm{m}=\mathrm{CWmax}$;

- $\mathrm{n}$ : minimum size of $\mathrm{CW}, \mathrm{n}=\mathrm{CWmin}$.

We define that the probability of RTSn/CTSn transmission failure is $P$. The wireless channel is assumed to be well, so the failing reason of nodes access channel is mainly data collision. Additionally, when nodes density reaches a certain level, $\mathrm{P}$ is a constant in theory. In this paper, we make a model about adaptive back-off mechanism. As shown in Fig. 7, in the Markov chain, the states of nodes are defined by pairs of integers $\{\mathrm{a}(\mathrm{t}), \mathrm{b}(\mathrm{t})\}$ as follows.

Before control frame is sent:

$$
P\{c, k \mid c, k+1\}=1, c \in[n, m], k \in[0, c-2] .
$$

$\mathrm{P}\{\mathrm{c}, \mathrm{k} \mid \mathrm{c}, \mathrm{k}+1\}$ represents the transition probability from the state $\{\mathrm{a}(\mathrm{t})=\mathrm{c}, \mathrm{b}(\mathrm{t})=\mathrm{k}+1\}$ to the state $\{\mathrm{a}(\mathrm{t}+1)=\mathrm{c}, \mathrm{b}(\mathrm{t}+1)=\mathrm{k}\}$, Eq. (1) represents that the random timer minus 1 in the beginning of moment $t$.

After control frame is sent:

$$
\begin{aligned}
& P\{c, k \mid c, 0\}=(1-P) / c, c \in(n, m], k \in[0, c-1] . \\
& P\{c, k \mid c, 0\}=P / i, i \in[n, m), k \in[0, c-1] . \\
& P\{m, k \mid m, 0\}=P / m, k \in[0, m-1] . \\
& P\{n, k \mid n, 0\}=(1-P) / n, k \in[0, n-1] .
\end{aligned}
$$

Eq. (2) represents that $\mathrm{CW}$ cur randomly increases after a successful transmission, then $\mathrm{CW}$ value is randomly selected from [0, c-1]. Eq. (3) represents that CWcur randomly decreases after a failing transmission, then $\mathrm{CW}$ value is randomly selected from [0, c-1]. Eq. (4) represents that $\mathrm{CW}$ can only be randomly selected from [ $0, \mathrm{~m}-1]$, when CWcur reaching the maximum size after a transmission failure. Eq. (5) represents that $\mathrm{CW}$ can only be randomly selected from [ $0, n-1]$, when CWcur reaching the minimum size after a successful transmission.

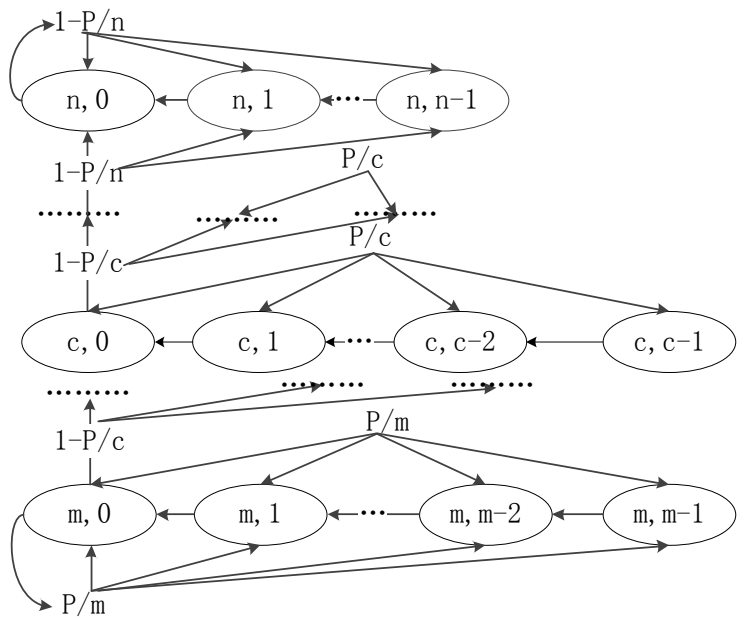

Fig. 7: Markov model of back-off process in scheduling phase.

According to the ergodicity of Markov chain, we know stationary distribution of $\{a(t), b(t)\}$ is limit distribution, so we define the limit distribution:

$$
\begin{aligned}
& b_{c, k}=\lim _{t \rightarrow \infty} P\{a(t)=c, b(t)=k\}, \\
& i \in[n, m], k \in[0, c-1] .
\end{aligned}
$$

Eq. (6) is the limit distribution of $\{a(t), b(t)\}$, so $b_{c, 0}$ has the following properties: 


$$
\begin{aligned}
& b_{c 1,0} * P=b_{c 2,0} \rightarrow b_{c, 0}=P^{d} * b_{n, 0}, \\
& c 1 \leq c 2, c \in[n, m], d \in[1,+\infty) . \\
& b_{n, 0} * P^{d 1}=(1-P)^{d 2} * b_{c, 0} \rightarrow b_{c, 0}=\frac{P^{d}}{1-P} * b_{n, 0}, \\
& c \in[n, m], d \in[1,+\infty) . \\
& \sum_{c=n}^{c=m} b_{c, 0}=\frac{1+P^{d 1}+\ldots . .+P^{d n}}{1-P} * b_{n, 0}, d \in[1,+\infty) .
\end{aligned}
$$

According to the regularity of the Markov chain, we get $\mathrm{b}_{\mathrm{c}, \mathrm{k}}$ :

$$
b_{c, k}=\frac{c-k}{c} *\left\{\begin{array}{c}
(1-P) * b_{n, 0}, c=n \\
P * b_{c, 0}, c \in(n, m) \\
(1-P)^{*} b_{c, 0}, c \in(n, m) \\
P^{*} b_{m, 0}, c=m
\end{array} .\right.
$$

Put Markov normalization, all $b_{c, k}$ is represented by $b_{n, 0}$ and P:

$$
\begin{aligned}
& b_{n, 0}=\frac{2}{\left[\sum_{c=n}^{c=m} * P^{d 1} * c+\frac{P^{d 2}}{1-p} * m+\frac{1}{1-P}\right]}, \\
& c \in[n, m], d \in[1,+\infty) .
\end{aligned}
$$

We define that the probability of RTSn/CTSn transmission success is q. When network is stable, $\mathrm{q}$ is equal to the sum of nodes' successful transmission probabilities in each time slot.

$$
q=\sum_{c=n}^{c=m} b_{c, 0}(1-P)=\frac{b_{n, 0}}{1-P}(1-P)=b_{n, 0} .
$$

In this case, if there are $w$ nodes in the network, the probability of control frame transmission failure is equal to the probability of the remaining $(\mathrm{w} / \mathrm{m})-1$ nodes sending data, then P:

$$
P=1-(1-q)^{\frac{w}{m}-1} \text {. }
$$

The data transmission is completed by the relay nodes' transmission, so packet delivery ratio of SQ-MAC is determined by the packet delivery rate of single node. In theory, collision-free data exchange in scheduling time slot, after completing time slots scheduling. So the packet delivery ratio of data that nodes can achieve is determined by the packet delivery rate of RTSn/CTSn control frame. For different traffic classes, since RTdown $>$ NRTdown, RTup<NRTup, RT's d is greater than NRT's d in CW increase process, and RT's d is less than NRT's d in the $\mathrm{CW}$ decrease process, so PRT <PNRT, that is, the packet delivery ratio of RT is greater than NRT.

\subsection{Latency}

In this section, we assume that nodes realize collision-free in data phase, so the end-to-end latency is equal to the transmission time of data packets, which from successfully entered the source node's data queue to successfully received by the destination node.

For RT, the latency expression is as shown in Eq. (14), where $T_{\text {queue-RT }}$ is the queue latency of $R T, z$ is the upper limit of RTSn retransmission, $\mathrm{T}_{\text {sync }}$ is the time of status update and synchronization in synchronization phase, $T_{\mathrm{sc}}$ is the time length of scheduling phase, $\mathrm{T}_{\text {cycle }}$ is the time length of period.

$$
\begin{aligned}
& T_{R T}=\left(T_{\text {sync }}+T_{s c}+2 T_{s t}\right) \sum_{j=0}^{j=z}\left(1-P_{R T}\right) P_{R T}{ }^{j} \\
& +\sum_{j=0}^{j=z} P_{R T}^{j}\left(1-P_{R T}\right) j^{*} T_{\text {cycle }}+T_{\text {queuе }-R T} .
\end{aligned}
$$

For NRT, the latency expression is as shown in Eq. (15), where $\mathrm{T}_{\text {queue-NRT }}$ is the queue latency of NRT.

$$
\begin{aligned}
& T_{N R T}=\left(T_{\text {sync }}+T_{s c}+(S-C+2) * T_{s t}\right) * \sum_{j=0}^{j=z}\left(1-P_{N R T}\right) P_{N R T}^{j} \\
& +\sum_{j=0}^{j=z} P_{N R T}^{j}\left(1-P_{N R T}\right) j * T_{\text {cycle }}+T_{\text {queue }-N R T} .
\end{aligned}
$$

Since SQ-MAC uses priority queue mechanism in the process of data scheduling, RT can more easily be sent than NRT, that is, $\mathrm{T}_{\text {queue-RT }}<\mathrm{T}_{\text {queue-NRT. }}$ Additionally, PRT $<$ PNRT, it is found that $\mathrm{T}_{\text {latency-RT }}<\mathrm{T}_{\text {latency-NRT }}$ in conclusion.

\subsection{Energy Consumption}

The related scholars usually use listening time of nodes to measure the energy consumption of network. This paper also uses it to measure the energy consumption of SQMAC.

In the ideal condition, we analyze the listening time of SQMAC in Eq. (16), where $T_{\text {backoff }}$ is the time of channel contention in scheduling phase.

$$
\text { Time }^{\text {ideal }}=T_{\text {sync }}+T_{\text {backoff }}+T_{\text {st }} .
$$

When network load is light, there is $T_{\text {backoff }}=0, T_{s t}=T_{\text {hall }}$. Therefore, the listening time is:

$$
\text { Time } e^{\text {light }}=T_{\text {sync }}+\text { Thall. }
$$

When the network is saturated, we further analyze the listening time of SQ-MAC, as shown in Eq. (18).

$$
\begin{aligned}
& \text { Time }=\text { Time }^{\text {ideal }} \sum_{j=0}^{j=z}(1-P) P^{j} \\
& +\sum_{j=0}^{j=z} j(1-P) P^{j}\left(T_{\text {sync }}+T_{\text {backoff }}\right) .
\end{aligned}
$$

After analysis, we found the energy consumption of different traffic classes are not the same. When the load is light, Time $_{\mathrm{RT}}=$ Time $_{\mathrm{NRT}}$, because the idle listening time is 
Thall. When the load is saturation, we can see that Time $_{\mathrm{RT}}<$ Time $_{\mathrm{NRT}}$, because $\mathrm{P}_{\mathrm{RT}}<\mathrm{P}_{\mathrm{NRT}}$.

\section{Simulation Results}

In order to further research the performance of SQ-MAC, we use network simulation software NS2 (Network Simulator version 2) to simulate SQ-MAC in a multi-hop crossover network topology. As shown in Fig. 8, the network topology is the orthogonal superposition of two multi-hop chain networks, and it is mainly used to test the MAC capacity for avoiding or decomposing data collision. Moreover, we make a comparison with S-MAC and DQMAC.

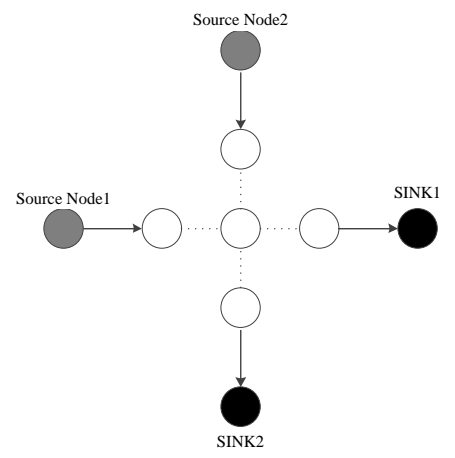

Fig. 8: Multi-hop crossover network topology in simulation.

In the simulation, the source nodes generate CBR traffic, and continually send to SINK node. For SQ-MAC, one source node sends RT, and another node sends NRT. We assume that the two source nodes simultaneously send data packets and network's hops (path length) are 6.

Table 1: Partial Simulation Parameter Setting

\begin{tabular}{|c|c|}
\hline Parameter & Value \\
\hline transmission distance & $250 \mathrm{~m}$ \\
\hline data packet length & $50 \mathrm{Byte}$ \\
\hline simulation time & $1000 \mathrm{~s}$ \\
\hline routing & DSR \\
\hline sending power & $22.6 \mathrm{mw}$ \\
\hline received power & $15.1 \mathrm{mw}$ \\
\hline listening power & $15 \mathrm{mw}$ \\
\hline sleeping power & $0.5 \mathrm{mw}$ \\
\hline CWmax & 63 \\
\hline CWmin & 15 \\
\hline
\end{tabular}

Firstly, we simulate the effect of SQ-MAC parameters on QoS-aware function, wherein the packet arrival interval of nodes is set to $5 \mathrm{~s}$.
Fig. 9 shows the effect of time slots ratio on the latency of different traffic classes, we set RTdown $=$ NRTup $=0.6$, RTup $=$ NRTdown $=0.2, \quad$ DW $(\mathrm{NRT})=1, \mathrm{DW}(\mathrm{RT})=0.3$ and $\mathrm{S}=6$. When $\mathrm{C}$ increased from 1 to 6 , the latency of NRT is gradually close to RT. While NRT won't be better than RT because the factors of $\mathrm{CW}$ and data queue.

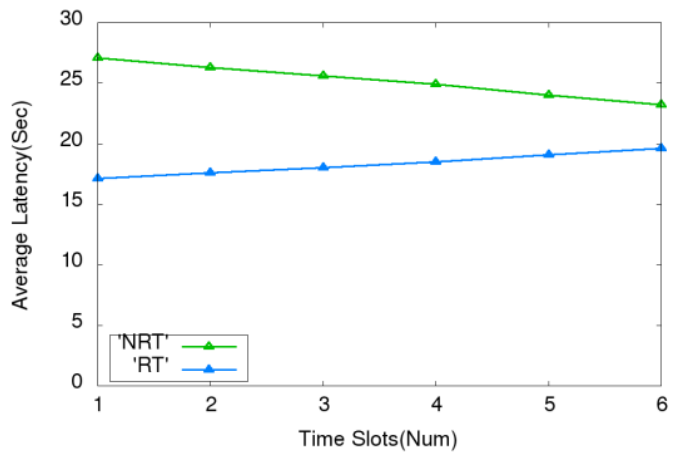

Fig. 9: Effect of time slots ratio on the latency.

Fig. 10 shows the effect of $\mathrm{CW}$ parameters on the $\mathrm{CW}$ size of different traffic classes, we set $D W(N R T)=1$, $\mathrm{DW}(\mathrm{RT})=0.3, \mathrm{~S}=6, \mathrm{C}=3$, RTdown $=0.6$ and $\mathrm{RTup}=0.2$. When NRTdown increased from 0.1 to 0.6 , and NRTup decreased from 0.6 to 0.1 simultaneously, the CW size of NRT has gradually decreased, while RT maintains a relatively stable value.

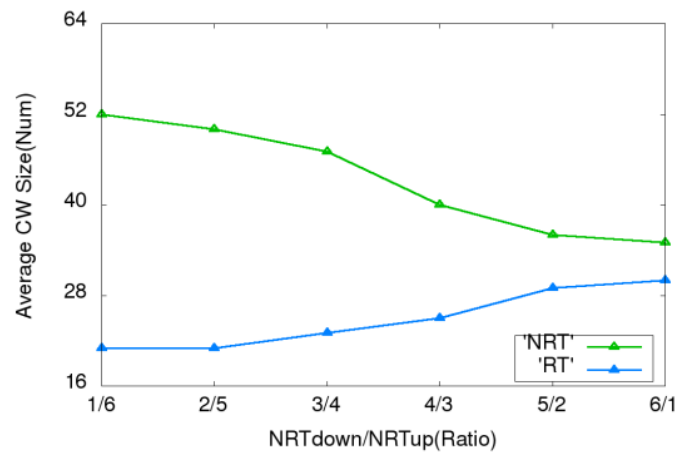

Fig. 10: Effect of CW parameters on the $\mathrm{CW}$ size.

Fig. 11 shows the effect of queue weight on the latency of different traffic classes, we set RTdown $=$ NRTup $=0.6$, RTup $=$ NRTdown $=0.2, C=3, S=6$ and DW(NRT) $=1$. When $\mathrm{DW}(\mathrm{RT})$ increased from 0.1 to 1 , the latency of NRT is gradually close to RT, while NRT is always higher than RT. 


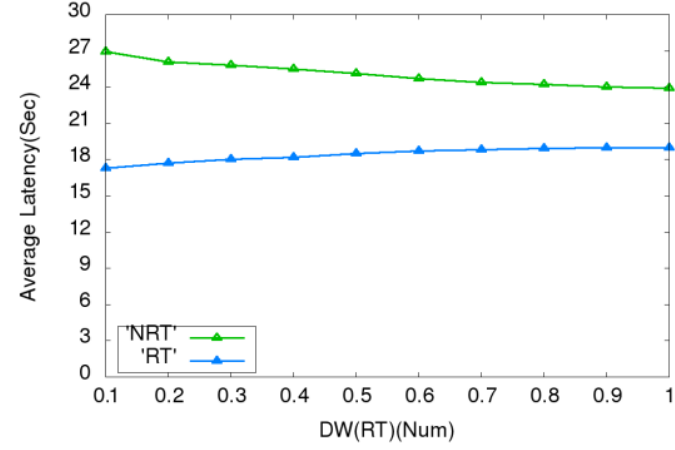

Fig. 11: Effect of queue weight on the latency.

We simulate the network performance of all protocols when the packet arrival interval increasing from $1 \mathrm{~s}$ to $10 \mathrm{~s}$, which shows that the load is from high to low, Additionally, for SQ-MAC, we set RTdown=NRTup=0.6, RTup $=$ NRTdown $=0.2, \mathrm{DW}(\mathrm{NRT})=1, \mathrm{DW}(\mathrm{RT})=0.3, \mathrm{~S}=6$ and $\mathrm{C}=3$. For $\mathrm{S}-\mathrm{MAC}$, both source nodes send data packets with the same priority level. For DQ-MAC, we reset all MAC parameters to the default values, wherein one source node sends RT, and another node sends BE [5].

Fig. 12 shows the variation of packet delivery ratio. When packet arrival interval is low, because SQ-MAC can reduce the probability of data collision in the process of data exchange, the packet delivery ratio of SQ-MAC is obviously better than other MAC protocols. Compared with SQ-MAC-NRT, there're more time slots can be used for SQ-MAC-RT to send data packets, so the packet delivery ratio of SQ-MAC-RT has increased by nearly $15 \%$. With the increase of packet arrival interval, network load is gradually lower and the packet delivery ratios of all protocols ultimately reach a constant level.

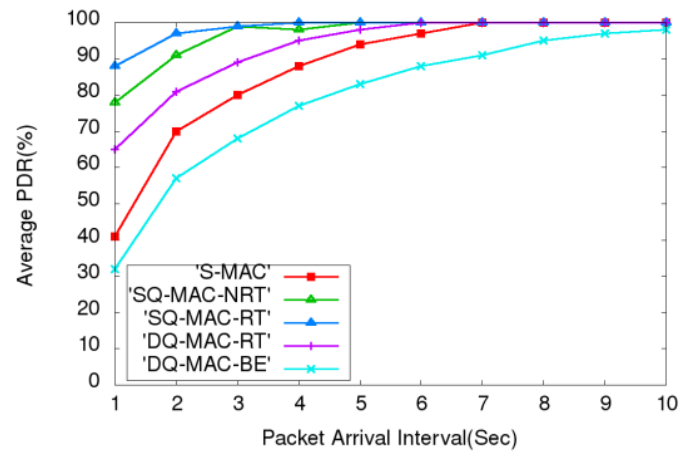

Fig. 12: Average packet delivery ratio with the load varies.

Fig. 13 shows the variation of latency. Because the process of SQ-MAC data exchange is dispersed to the entire data phase, SQ-MAC can send more data packets in the same time compared with S-MAC and DQ-MAC, so the latency of SQ-MAC is lower than them. Compared with SQ-MAC-
NRT, the probability of SQ-MAC-RT access channel is higher, the number of available time slots is more, and the time slots is more forward, so the latency of SQ-MAC-RT is reduced by nearly $25 \%$.

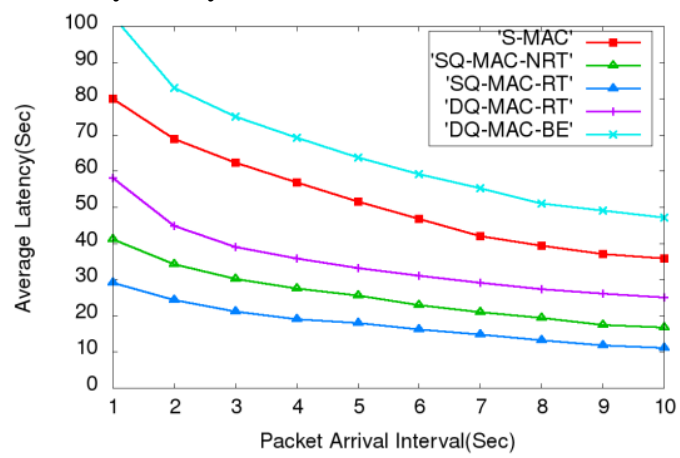

Fig. 13: Average latency with the load varies.

Fig. 14 shows the variation of total energy consumption. SQ-MAC makes unrelated nodes turn to sleep during network data exchange, thus reducing idle listening time of nodes. The energy consumption of SQ-MAC is reduced by nearly $22 \%$ when compared to S-MAC, and reduced by nearly $20 \%$ when compared to DQ-MAC.

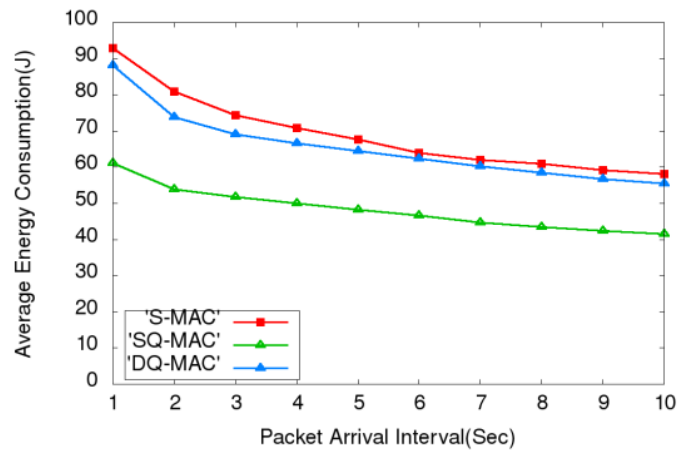

Fig. 14: Average energy consumption with the load varies.

\section{Conclusions}

This paper proposes a QoS-aware MAC protocol, SQMAC. The protocol uses slot-scheduling mechanism, adaptive back-off mechanism, virtual multi-queue and fair scheduling mechanism to make the MAC protocol acquire the advantages of low collision, low power consumption, and adaptation on the premise of achieves service differentiation. Simulation results show that, compared with the S-MAC and DQ-MAC, SQ-MAC has a better network performance in term of data delivery ratio, latency and energy consumption. Additionally, the high priority traffic consumes more network resources compared with the low priority traffic. 


\section{Acknowledgments}

This work is supported by the National Key Technology R\&D Program of China (2015BAF32B04-3), the Joint Funds of the National Natural Science Foundation of China (Grant no. U1404615), the Key Science and Research Programin University of Henan Province (16A460018), the Project of Basic and Advanced Technology Research of Henan Province of China (152300410081), the Program for Innovative Research Team (in Science and Technology) in University of Henan Province (15IRTSTHN008), Open Funds of State Key Laboratory of Millimeter Waves (Grant no. K201504), and China Post doctoral Science Foundation (Grant no. 2015M571637).

\section{References}

[1] I. F. Akyildiz, W. Su and Y. Sankarasubramaniam, "Wireless Sensor Networks: a Survey," Computer Networks, vol. 38, no. 4, 2002, pp. 393-422.

[2] M. A. Yigitel, O. D. Incel and C. Ersoy, "QoS-aware MAC protocols for wireless sensor networks: A survey", Computer Networks, vol.55, no.4, 2011, pp.1982-2004.

[3] Bhattacharya D, Krishnamoorthy R. "Power Optimization in Wireless Sensor Networks", International Journal of Computer Science Issues, 2011, vol.8, no.5.

R. T. Matani and T. M. Vasavada, "A Survey on MAC Protocols for Data Collection in Wireless Sensor Networks," International Journal of Computer Applications, vol. 114, no. 6, 2015, pp. 4-7.

[4] N. Saxena, A. Roy and J. Shin, "Dynamic duty cycle and adaptive contention window based QoS-MAC protocol for wireless multimedia sensor networks," Computer Networks, vol. 52, no. 13, 2008, pp. 2532-2542.

[5] J. He, G.W. Bai and L. Cao, "DQ-MAC: A Diffserv-based MAC Mechanism in Wireless Sensor Networks," Computer Science, vol. 37, no. 12, 2010, pp.30-34.

[6] P. Suriyachai, U. Roedig and A. Scott, "Implementation Of A Mac Protocol For QoS Support In Wireless Sensor Networks," in Proceedings of 7th Annual IEEE International Conference on Pervasive Computing and Communications, Galveston, 2009, pp. 1-6.

[7] L. Zhang, L. Da-Shuang, J. B. Mao and Z.Y. Jing, "A TDMA MAC Protocol Supporting QoS in Ad Hoc Network," Communications Technology, vol. 47, no. 10, 2014, pp.1162-1166.

[8] I. Slama, B. Shrestha and B. Jouaber, "A hybrid MAC with prioritization for wireless sensor networks," in Proceedings of 33rd IEEE Conference on Local Computer Networks (LCN), Montreal, 2008, pp. 274-281.

[9] J. Liu, Z. Wang, Y. Huo and Y. Wang, "A Hybrid MAC Protocol with QOS Guarantee in Ad hoc Network," in Proceedings of International Conference on Computer Science and Information Technology, Springer India, 2014, pp. 269-277.

[10]W. Ye, J. Heidemann and D. Estrin, “An energy-efficient MAC protocol for wireless sensor networks," in Proceedings of IEEE INFOCOM 2002, New York, 2002, pp. 1567-1576.
[11]W. Ye, J. Heidemann and D. Estrin, "Medium Access Control With Coordinated Adaptive Sleeping for Wireless Sensor Networks," IEEE/ACM Transactions on Networking, vol. 12, no. 3, 2004, pp. 493-506.

[12]A. K. Jacob and L. Jacob, "Energy Efficient MAC for QoS Traffic in Wireless Body Area Network," International Journal of Distributed Sensor Networks, vol. 2015, 2015, pp. $1-12$.

[13]Z. Hamid and F. B. Hussain, "QoS in Wireless Multimedia Sensor Networks: A Layered and Cross-Layered Approach," Wireless Personal Communications An International Journal, vol. 75, no. 1, 2014, pp. 729-757.

[14]B. Cheng, L. Ci and C. Tian, "Contention Window-Based MAC Protocol for Wireless Sensor Networks," in Proceedings of 12th IEEE International Conference on Dependable, Autonomic and Secure Computing, Dalian, 2014, pp. 479-484.

[15]X. Fafoutis, C. Orfanidis and D.Nicola, "Altruistic Backoff: Collision Avoidance for Receiver-Initiated MAC Protocols for Wireless Sensor Networks," International Journal of Distributed Sensor Networks, vol. 37, no. 1, 2014, pp. 4-4.

[16]C. Min and Y. I. Eom, "Integrating Lock-Free and Combining Techniques for a Practical and Scalable FIFO Queue," Parallel and Distributed Systems, IEEE Transactions on, vol. 26, no. 7, 2015, pp. 1910-1922.

Guoqiang Zheng Received the Ph.D. degree in Communication and information systems professional from Xi'an Jiaotong University, China, 2008. He is a professor at Henan University of Science and Technology on College of Electronic Information Engineering, China. His research interests include wireless communication technology, network communication protocol and software radio theory.

Yaru Sun Received the B.S. degree in Henan University of Science and Technology, Luoyang, China in 2014. She is currently working towards M.S. degree in Henan University of Science and Technology, Luoyang, China. Her research interests include WSNs and MAC Protocol.

Bingwu Kang Received the B.S. degree in Luoyang Normal University, Luoyang, China in 2013. And received the M.S. degree in Henan University of Science and Technology, Luoyang, China in 2016.

Huahong Ma Received her master degree in Signal and Information Processing in July 2005 at Yunnan University, China. Now, she is a Ph.D. candidate in Control Science and Engineering at Henan University of Science and Technology. Her main research interests are Crowd Sensing Network and Internet of Things.

Jishun Li Received the Ph.D. degree in Mechanical Manufacture and Automation from Shanghai Jiaotong University, China, 1996. $\mathrm{He}$ is a professor at Henan University of Science and Technology on College of Mechatronics Engineering, China.

Yuting Wang Received the B.S. degree in Henan University of Science and Technology, Luoyang, China in 2015. She is currently working towards M.S. degree in Henan University of Science and Technology, Luoyang, China. 